\title{
Driving Potentials of Heat and Mass Transport in Porous Building Materials: A Comparison Between General Linear, Thermodynamic and Micromechanical Derivation Schemes
}

\author{
Max Funk • Karim Ghazi Wakili
}

Received: 31 January 2007 / Accepted: 11 June 2007 / Published online: 20 July 2007

(C) Springer Science+Business Media B.V. 2007

\begin{abstract}
In systems of coupled transport processes the question of the appropriate driving potentials is a point of discussion. In this article, three different approaches to derive models for transport currents are systematically compared. According to a general linear approach, an arbitrary full set of independent state variables and material properties is sufficient to describe any transport current. This approach is derived here from a symmetry principle. Thermodynamic and micromechanical approaches are more complex and even less general, but they allow additional statements about the transport coefficients and they reduce the number of transport processes. In the thermodynamic approach the additional information stems from the calculation of the entropy production rate; the micromechanical approach involves a microphysical model of the considered porous system. As a practical example, the three derivation schemes are applied to the often-encountered case of non-hysteretic heat and moisture transport in homogeneous building materials. It is shown, how the general state variables of a porous system are reduced to only two. Then from the general linear approach it can be seen, that all equations for the moisture transport current using a main driving potential (e.g. moisture content, vapour pressure, chemical potential) and an independent secondary driving potential (e.g. temperature, liquid pressure) are equivalent, without recurrence to the thermodynamic or micromechanical approach. However, the transport coefficients are arbitrary phenomenological functions depending on the two state variables. Based on a literature survey it is shown, which additional statements can be made in the thermodynamic and in the micromechanical approach. The latter yields the pressure-driven model (vapour and liquid pressure as the two driving potentials). Finally it is shown, what is to be expected, if in more complex systems the number of state variables increases.
\end{abstract}

Keywords Moisture diffusion - Moisture conduction - Coupled heat and moisture . Thermodiffusion - State variables · Building materials · Driving potentials $\cdot$ Linear models

M. Funk $(\bowtie) \cdot$ K. G. Wakili

Building Technologies, Swiss Federal Laboratories for Materials Testing and Research, EMPA, Ueberlandstrasse 129, 8600 Duebendorf, Switzerland

e-mail: max.funk@empa.ch 


\section{Introduction}

\subsection{General Introduction}

Transport processes in porous building materials have always been the subject of intensive investigations, since it is of fundamental importance to improve the energetic and hygric behaviour of building constructions, in order to ensure a comfortable indoor climate, to save energy and to avoid damages induced by moisture, salt efflorescence and freezing.

Different models for the coupled heat, air, moisture and salt transport have been developed and incorporated into various software programs used in the field of porous building materials and in the closely related field of wetting and drying of soils. Regarding the heat and moisture transport in building materials there are WUFI, based on Kuenzel (1994), Delphin4 (Grunewald 1997), CHAMPS/Delphin 5 (Nicolai et al. 2007), (Grunewald et al. 2006), MATCH (Pedersen 1992), finally a MATLAB implemetation (Sasic Kalagasidis 2004) based on Hagentoft et al. (2004). In the field of soil science there are Hydrus3D (Simunek et al. 2003) and MACRO (Jarvis 1994). Finally, there is the program package FEMLAB (COMSOL 2005), incorporating various models for saturated and unsaturated moisture flow in its "Earth science module", including the Richard's equation system (unsaturated Darcy law), and the Brinkman equations (saturated Darcy and Navier-Stokes).

The history of models for the mass currents starting with the famous Darcy Model (Darcy 1856) has been reviewed by Lage (Ingham 1998). The models for saturated and unsaturated moisture transport as well as coupled heat and moisture transport have been further investigated e.g. by Whitaker (1986a), Hassanizadeh (1990), Gray (1991), and Grunewald (1997).

To a major part, the complexity of a system can be characterised by its independent state variables. In the introductory part of the article, it will be shown schematically, which state Variables arise in a complex multi-phase and multi-domain porous system and under which assumptions these can be reduced step by step to arrive at the case of two variables only (energy and mass content), which describe the simple, but commonly described case of nonhysteretic heat and moisture transport in homogeneous materials. Our study will begin with an introductory overview of models for the moisture current describing this situation.

In the main part of the article, first a general linear approach will be derived, which will be applied to the example models subsequently. In the following, the properties of more complex derivation schemes found in literature, the thermodynamic and the micromechanical approach, will be reviewed. This will be done also first in general, then applied to the example case. The additional information content given by these approaches will be determined. Finally, the generalisation to systems with more than two state variables will be discussed.

\subsection{Models for Moisture Currents in the Non-hysteretic Heat and Moisture Transport}

The moisture current $j^{M_{w+v}}$ penetrating a porous building material is in linear approximation given by a sum of products of conductivities and gradients:

$$
j^{M_{w+v}}=-\Sigma_{i} K_{i}^{\vec{\Phi}} \cdot \frac{\mathrm{d} \Phi_{i}}{\mathrm{~d} x}
$$

$M_{w}$ and $M_{v}$ are liquid water mass and water vapour mass, respectively which combine to the total moisture mass given by $M_{w}+M_{v} \equiv M_{w+v}$. 
$\vec{\Phi}=\left(\Phi_{1}, \ldots, \Phi_{i}, \ldots, \Phi_{n}\right)$ is the vector of driving potentials used to describe the coupled transport. $K_{i}^{\vec{\Phi}}$ is the moisture conductivity corresponding to the gradient of $\Phi_{i}$ and to the chosen set of driving potentials $\vec{\Phi}$. Note, that a general conductivity coefficient $K$ depends first on the transported quantity, second on the chosen gradient $\mathrm{d} \Phi_{i} / \mathrm{d} x$ (abbreviated by $\partial \Phi_{i}$, or just " $\mathrm{i}$ ") and third on the whole set of driving potentials $\vec{\Phi}$, because in Eq. 1 it is stated, that the part of the current, which stems from $\mathrm{d} \Phi_{i} / \mathrm{d} x$, has to be distinguished from the other parts, which stem from $\mathrm{d} \Phi_{j} / \mathrm{d} x, j \neq i$. This becomes clear from the explicit expression for $K$ which is given below, Eq. 19. It is also shown in Eq. 30. To simplify the notation, the dependence of the transported quantity has been omitted, since in the text below only moisture transport will be considered in the practical example.

If liquid water and water vapour transport are treated separately (the so-called "phasedivided transport"), different driving sets of driving potentials can be used for the liquid and water vapour transport, respectively, and (1) is replaced by:

$$
\begin{aligned}
& j^{M_{w}}=-\Sigma_{i} K_{i}^{\vec{\Phi}^{w}} \cdot \frac{\mathrm{d} \Phi_{i}^{w}}{\mathrm{~d} x} ; j j^{M_{v}}=-\Sigma_{i} K_{i}^{\vec{\Phi}^{v}} \cdot \frac{\mathrm{d} \Phi_{i}^{v}}{\mathrm{~d} x} \\
& j^{M_{w+v}}=j^{M_{w}}+j^{M_{v}}
\end{aligned}
$$

The pressure-driven model $\left(\operatorname{grad} p_{v}, \operatorname{grad} p_{c}\right)$ : The moisture transport process according to the pressure-driven model is defined in various sources, e.g. Grunewald (1997), Hagentoft et al. (2004) and prEN15026 (2006). In the general notation of (2) and omitting air and salt transport, it reads:

$$
\begin{aligned}
& j^{M_{w+v}}=j^{M_{w}}+j^{M_{v}}=K_{p_{c}}^{\vec{\Phi}^{w, \text { pressure }}} \cdot \frac{\mathrm{d} p_{c}}{\mathrm{~d} x}+K_{p_{v}}^{\vec{\Phi}^{v, \text { pressure }}} \cdot \frac{\mathrm{d} p_{v}}{\mathrm{~d} x} \\
& \vec{\Phi}^{w, \text { pressure }}=\left(p_{c}\right) ; \quad \vec{\Phi}^{v, \text { pressure }}=\left(p_{v}\right)
\end{aligned}
$$

$p_{v}$ is the water vapour pressure in the gaseous phase and $p_{c}$ is the capillary pressure given by the difference of liquid and gaseous pressure:

$$
p_{c} \equiv p_{\ell}-p_{g}
$$

In case of constant gas pressure ( $p_{g}=$ const), the liquid pressure and capillary pressure differ only by a constant, hence their gradients are identical. There is only one driving potential for each phase ( $p_{c}$ and $p_{v}$, respectively).

The liquid diffusivity/vapour pressure model ( $\operatorname{grad} p_{v}, \operatorname{grad} m_{w+v}$ ): This moisture transport equation uses the vapour pressure gradient as driving potential for the vapour phase and the moisture content gradient for the liquid phase. With respect to the liquid current, it is a diffusion model. With respect to the vapour current it is a pressure model. It is documented in (Kuenzel 1995), Eqs. 11, 13, 35, also in (Holm 2001), Eq. 7. In the notation of (2) it reads

$$
\begin{aligned}
& j^{M_{w+v}}=j^{M_{w}}+j^{M_{v}}=-K_{m_{w+v}}^{\vec{\Phi}^{w, \text { diffusion }}} \cdot \frac{\mathrm{d} m_{w+v}}{\mathrm{~d} x}-K_{p_{v}}^{\vec{\Phi}^{v, \text { pressure }}} \cdot \frac{\mathrm{d} p_{v}}{\mathrm{~d} x} \\
& \vec{\Phi}^{w, \text { diffusion }}=\left(m_{w+v}\right) ; \quad \vec{\Phi}^{v, \text { pressure }}=\left(p_{v}\right)
\end{aligned}
$$

$m_{w+v}=M_{w+v} / V_{\mathrm{REV}}$ is the water mass per volume of porous medium. Again, there is only one driving potential for each phase.

The diffusivity model $\left(\operatorname{grad} m_{w}\right.$, grad $\left.T\right)$ : In the pure diffusivity moisture transport equation, particles flow from high moisture to low moisture regions. No phase-divided transport 
is considered. According to Eq. (1), the moisture current will read:

$$
\begin{aligned}
& j^{M_{w+v}}=-K_{m_{w+v}}^{\vec{\Phi}^{\text {diffusion }}} \cdot \frac{\mathrm{d} m_{w+v}}{\mathrm{~d} x}-K_{T}^{\vec{\Phi}^{\text {diffusion }}} \cdot \frac{\mathrm{d} T}{\mathrm{~d} x} \\
& \vec{\Phi}^{\text {diffusion }}=\left(m_{w}, T\right)
\end{aligned}
$$

In this case, we have two driving potentials and no distinction between the phases, since they are in equilibrium. The main driving force is the moisture content $m_{w+v}=M_{w+v} / V_{R E V}$. The temperature $T$ accounts for the effect of thermodiffusion.

The Glaser model $\left(\operatorname{grad} p_{v}\right)$ : The Glaser approach (EN ISO 13788 2001) describes the total moisture transport in terms of the vapour pressure gradient.

$$
j^{M_{w+v}}=-K_{p_{v}}^{\vec{\Phi}_{\text {Glaser }}} \cdot \frac{\mathrm{d} p_{v}}{\mathrm{~d} x} ; \quad \vec{\Phi}_{\mathrm{Glaser}}=\left(p_{v}\right)
$$

An expression for the moisture current having only one gradient though there are two independent state variables has a limited validity; in this case it must be restricted to cases of low relative humidity where the gaseous transport dominates. Note, that also in another respect the Glaser model is a simplification, since it does not solve the space- and time-dependent transport equation (8) below.

\subsection{System State Variables and Transport Equations}

The well-known transport equation for a conserved extensive quantity $W$ (e.g. inner energy, moisture) or its volume-specific form $w(\vec{x}, t)$ can be written in differential form:

$$
\frac{\partial}{\partial t}\left(W / V_{\mathrm{REV}}\right)=\frac{\partial}{\partial t} w(\vec{x}, t)=-\sum_{k \in\{x, y, z\}} \frac{\partial}{\partial x_{k}}\left(j_{k}^{W}(\vec{x}, t)\right)+\sigma^{W}(\vec{x}, t)
$$

$V_{\mathrm{REV}}$ is the volume of the so-called "representative volume element" (REV). It has been described by Bear (1991) and Whitaker (1986a). $\vec{j}^{W}$ denotes the transport current. $\sigma^{W}$ is the source/sink term defined by:

$$
\sigma^{W} \equiv \delta W_{\text {source }} /\left(V_{\mathrm{REV}} \cdot \delta t\right)
$$

It includes inflow from adjacent phases, from long range sources and from boundary effects. Each region $\alpha$, representing a great canonical ensemble, can be described by its inner energy $u_{\alpha}$, the masses $m_{\alpha, i}$ of all constituents $i$, the momentum $\vec{i}_{\alpha} \equiv \vec{I}_{\alpha} / V_{\mathrm{REV}}$ and the partial volume $\theta_{\alpha} \equiv V_{\alpha} / V_{\text {REV }}$ as state variables.

For a heat, air, moisture and salt transport model including multiple salt ions and nonequilibrium salt crystallisation according to Grunewald (1997) and Funk et al. (2006) the independent state variables become

$$
\vec{w}=\left(u, m_{a}, m_{w+v}, m_{s_{1}}, \ldots, m_{s_{\max }}, m_{c_{1}}, \ldots, m_{c_{\max }}\right) .
$$

$m_{a}, m_{w+v}, m_{s i}$, and $m_{c i}$ are the masses of dry air, moisture, dissolved and crystallised salts, respectively. The salt masses appear twice, since crystallised phase $(c)$ and dissolved phase $(s)$ are not assumed to be in mass exchange equilibrium.

The model of Hassanizadeh and Gray $(1990,1991)$ does not take component mixtures into account. Instead, it contains in addition the state variables of interfaces $\alpha \beta$ between the bulk phases, as well as of the contact line $\lg m$, where liquid $(l)$, gaseous $(g)$ and solid material phase $(m)$ are in contact with each other. From (Gray 1991), Eq. 5, it is possible to identify the vector of conserved extensive quantities (in the nomenclature used here): 


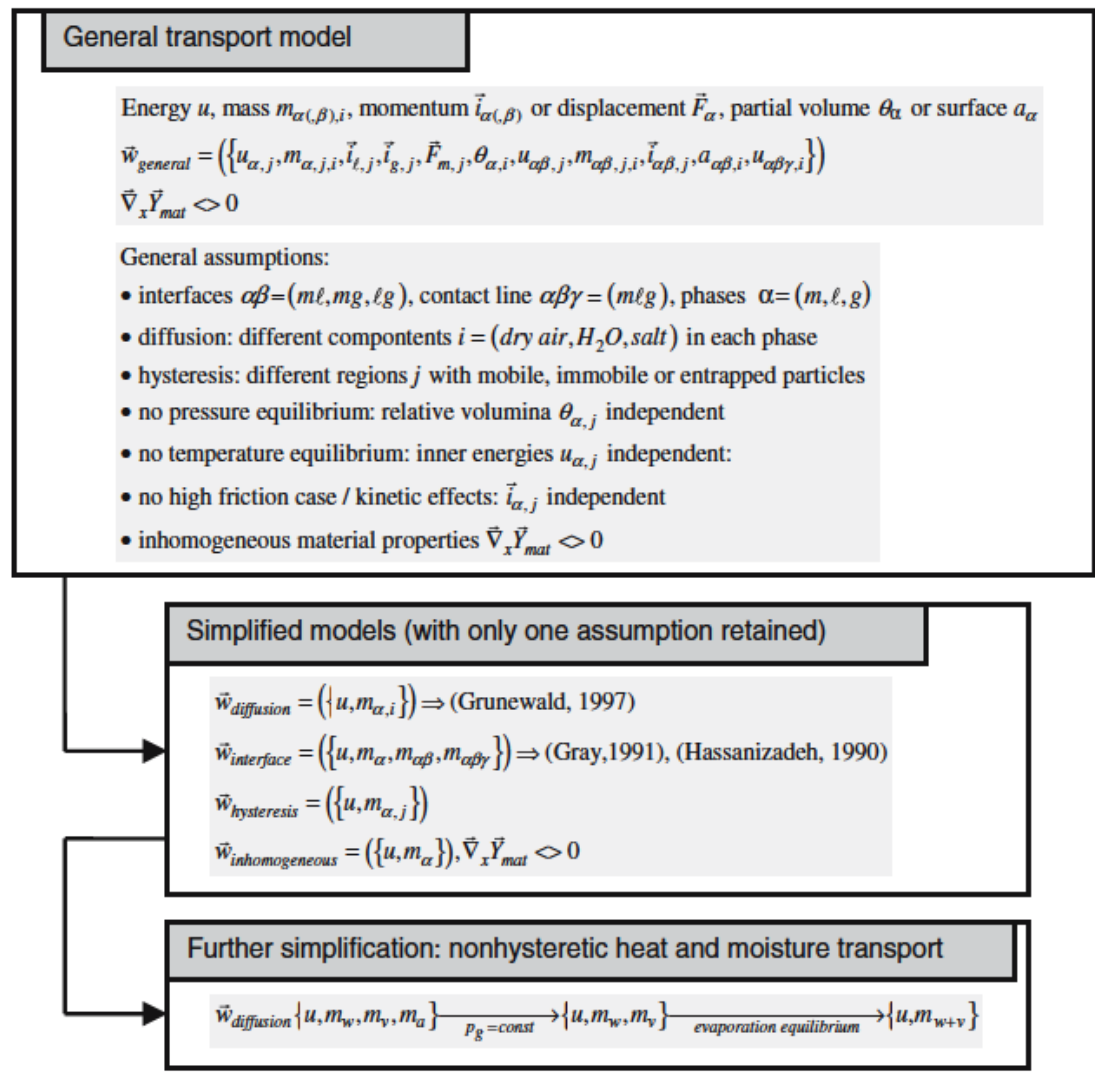

Fig. 1 State variables in the general model and reduction to the heat and moisture transport

$$
\vec{w}=\left(u_{\alpha}, \theta_{\ell}, \theta_{g}, m_{a}, m_{\ell}, m_{m}, \vec{i}_{l}, \vec{i}_{g}, \vec{F}_{m}, u_{\alpha \beta}, a_{\alpha \beta}, m_{\alpha \beta}, \vec{i}_{\alpha \beta}\right)
$$

The partial volumes $\theta_{\alpha}$, the specific interface areas $a_{\alpha \beta}=A_{\alpha \beta} / V_{\alpha \beta}$ and the specific momentums $\vec{i}_{\alpha}, \vec{i}_{\alpha \beta}$ are included as independent variables. In the solid phase $(m)$, the momentum vector has been replaced by the displacement vector $\vec{F}_{m}$, which denotes the displacement of the solid molecules from an assumed equilibrium position.

Combining the models (10) and (11), a very general expression for a full set of independent conserved extensive quantities of a REV can be found and subsequently be simplified down to the case of non-hysteretic heat and moisture transport. This is schematically shown in Fig. 1.

\section{The General Linear Approach}

\subsection{Derivation and Properties of the General Linear Approach}

In this section, it will be shown that the current $j^{W}$ of an arbitrary quantity $W$ depends only on a full set of independent state variables and space-dependent material properties and their gradients. If the gradients can assumed to be small of first order, then for an arbitrary choice 


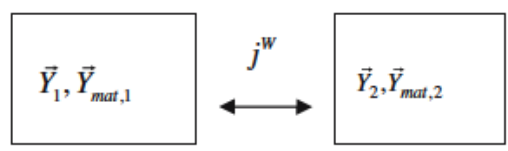

Fig. 2 Scalar flows between two reference volume elements

of state variables $\vec{Y}$ the current is always given as a linear combination of conductivities and the state variable gradients (See below, Eq. 20).

The derivation is as follows: Assume two reference volume elements $V_{\mathrm{REV}}$ with a scalar flow of $W$ in between as shown in Fig. 2. The $\vec{Y}_{1}, \vec{Y}_{2}$ vectors denote the state variables of the left and right volume elements, respectively. All other variable properties, e.g. inhomogeneous material properties, gravity potential etc. are denoted by $\vec{Y}_{\text {mat, } 1}, \vec{Y}_{\text {mat, } 2 \text {. If all properties }}$ would be identical, i.e. $\vec{Y}_{1}=\vec{Y}_{2}, \vec{Y}_{\text {mat, } 1}=\vec{Y}_{\text {mat, } 2}$, then there would be no preferred direction of a flow $j^{W}$. According to this symmetry principle, the flow $j^{W}$ must be zero. This property will be used in the derivation below. Generally, the current can be written as a function

$$
j^{W}=j^{W}\left(\vec{Y}_{1}, \vec{Y}_{2}\right)
$$

The dependency on $\vec{Y}_{\text {mat }}$ has been omitted here to simplify the notation. It will be reintroduced at the end of the derivation. The problem can be generalised to the continuous three-dimensional case: In this case the current $j^{W}$ at location $\vec{x}$ may depend on the state $\vec{Y}$ as well as on the states $\vec{Y}_{2}$ at all locations $\vec{x}_{2}$ in a region sufficiently close to location $\vec{x}$. Then the system states $\vec{Y}_{2}$ in distance $\Delta \vec{x}=\vec{x}_{2}-\vec{x}$ from the point $\vec{x}$ under consideration are given by

$$
\begin{aligned}
\vec{Y}_{2} & \approx \vec{Y}+\partial \vec{Y} / \partial x \cdot \Delta x+\partial \vec{Y} / \partial y \cdot \Delta y+\partial \vec{Y} / \partial z \cdot \Delta z \\
& =\vec{Y}+\operatorname{grad}(\vec{Y}) \cdot \Delta \vec{x}
\end{aligned}
$$

where the components of the gradient matrix are given in common nomenclatures by

$$
(\operatorname{grad}(\vec{Y}))_{i k} \equiv(D \vec{Y} / D \vec{x})_{i k} \equiv(\vec{\nabla} \vec{Y})_{i k} \equiv \partial Y_{i} / \partial x_{k} \equiv \partial_{k} Y_{i}
$$

If the system state in a small region around point $\vec{x}$ depends only on the state variables $\vec{Y}$ at the centre of the region and their gradients, then also the current depends only on these variables:

$$
\vec{j}^{W}=\vec{j}^{W}(\vec{Y}, \operatorname{grad} \vec{Y})
$$

If the gradients $\operatorname{grad}\left(Y_{i}\right)$ are all equal zero, this leads to identical states between the location under consideration and the neighbouring region and according to the above mentioned symmetry principle the currents must vanish:

$$
\vec{j}^{W}(\vec{Y}, \operatorname{grad} \vec{Y}=0)=0
$$


Treating the gradients in the system as small deviations from equilibrium, the current $\vec{j}^{W}$ can be Taylor expanded with respect to the $\operatorname{gradients} \operatorname{grad}(\vec{Y})$ :

$$
\begin{aligned}
\vec{j}^{W}(\vec{Y}, \operatorname{grad}(\vec{Y})) & =\left.\frac{D \vec{j}^{W}}{D(\operatorname{grad}(\vec{Y}))}\right|_{\operatorname{grad} \vec{Y}=0} \cdot \operatorname{grad}(\vec{Y}) \\
& =\Sigma_{i, k} \frac{\partial j_{k}^{W}}{\partial\left(\partial Y_{i} / \partial x_{k}\right)} \cdot \partial Y_{i} / \partial x_{k}
\end{aligned}
$$

In case of isotropic transport this simplifies to:

$$
\begin{aligned}
& j_{k}^{W}\left(\vec{Y}, \operatorname{grad}_{k} \vec{Y}\right)=-\Sigma_{i} K_{Y i}^{\vec{Y}}(\vec{Y}) \cdot \partial Y_{i} / \partial x_{k} \\
& k \in\{x, y, z\}
\end{aligned}
$$

where

$$
K_{Y_{i}}^{\vec{Y}}(\vec{Y}) \equiv-\frac{\partial j_{k}^{W}}{\partial\left(\operatorname{grad}_{k} Y_{i}\right)}=\frac{\partial j_{k}^{W}}{\partial\left(\partial Y_{i} / \partial x_{k}\right)}
$$

The result is in short form the general transport equation (1) for the flow of $W$ with conductivities $K(Y)$ and using the gradients of arbitrary state variables $\operatorname{grad}_{k} Y_{i}=\partial Y_{i} / \partial x_{k}$ as driving forces. The generalisation to the case of additional space-dependent properties $\vec{Y}_{\text {mat }}(\vec{x})$ is straightforward, as they can be treated in the same way as the state variables. Equation 18 becomes finally:

$$
\begin{aligned}
& j_{k}^{W}=-\Sigma_{i} K_{Y i}^{\vec{Y}, \vec{Y}_{\text {mat }}} \cdot \partial Y_{i} / \partial x_{k}-\Sigma_{j} K_{Y_{\text {mat }, j}}^{\vec{Y}, \vec{Y}_{\text {mat }}} \cdot \partial Y_{\text {mat }, j} / \partial x_{k} \\
& k \in\{x, y, z\}
\end{aligned}
$$

Note, that the term containing the gradient of the material properties $\operatorname{grad}_{k} Y_{\text {mat }}$ leads to important restrictions of the general linear approach: The external boundaries of the system and the internal boundaries between different materials can be considered as transition zones with steep gradients of the material properties. This explains the well-known fact, that in equilibrium arbitrary state variables (like water content) have also steep gradients at the internal boundaries and they are usually not chosen to describe transitions at the external boundaries. Also, in inhomogeneous materials with e.g. porosity as space-dependent material property, additional transport processes arise, for which the corresponding transport coefficients $K_{Y m a t}$ would be difficult to determine.

\subsection{Application to the Example Models for the Moisture Currents}

Assume that the state of the whole system is given by two independent state variables. The full local equilibrium assumption (i.e. no hysteresis) claims, that the heat and moisture distributes inside the REV in such a way, that the temperature and the chemical potential of the water molecules are the same everywhere. I.e. for each pair $\left(u, m_{w}\right)$, there is exactly one well-defined local equilibrium system state. On the other hand, for each well-defined equilibrium state a non-ambiguous characterisation via $\left(u, m_{w}\right)$ can be done. Therefore, the heat and moisture content is a valid set of independent state variables. Assuming invertible, strictly monotonous and differentiable relationships between energy and water content on the one hand and temperatures, pressures and chemical potentials on the other hand (i.e. between extensive variables $X_{i}$ and their associated thermodynamic potentials $P_{i}=\partial U / \partial X_{i}$ ), other sets of independent state variables may be constructed, containing pressures, temperatures and 
chemical potentials. The monotony of the functions $P_{i}\left(X_{j}\right)$ follows from thermodynamic stability conditions in combination with the assumption of no hysteresis; cf. e.g. Callen (1985), Chap. 8.2. However, whether these functions are strictly monotonous and differentiable and whether hysteresis occurs (especially in the high moisture region) must be examined carefully and will not be considered here.

As a first possibility, one may choose vapour pressure and temperature: $\left(p_{v}, T\right)$ as independent state variables and the water vapour mass $M_{v}$ as transported quantity. According to the general linear approach (20) the water vapour current is:

$$
\begin{aligned}
& \vec{\Phi}_{\text {pressure }}^{v}=\left(p_{v}, T\right) \\
& j^{M_{v}}=-K_{p_{v}}^{\vec{\Phi}_{\text {pressure }}^{v}} \cdot \frac{\mathrm{d} p_{v}}{\mathrm{~d} x}-K_{T}^{\vec{\Phi}_{\text {pressure }}^{v}} \cdot \frac{\mathrm{d} T}{\mathrm{~d} x}
\end{aligned}
$$

A second possibility is to choose capillary pressure and temperature $\left(p_{c}, T\right)$ as independent state variables and the liquid water mass $M_{w}$ as transported quantity. According to (20) the liquid water current is:

$$
\begin{aligned}
& \vec{\Phi}_{\text {pressure }}^{w}=\left(p_{c}, T\right) \\
& j^{M_{w}}=-K_{p_{c}}^{\vec{\Phi}_{\text {pressure }}^{w}} \cdot \frac{\mathrm{d} p_{c}}{\mathrm{~d} x}-K_{T}^{\vec{\Phi}_{\text {pressure }}^{w}} \cdot \frac{\mathrm{d} T}{\mathrm{~d} x}
\end{aligned}
$$

A third possibility is to choose water content and temperature $\left(m_{w}, T\right)$ as independent state variables and the liquid water mass $M_{w}$ as transported quantity. Once more, according to (20), an alternative formulation for the liquid water current is:

$$
\begin{aligned}
& \vec{\Phi}_{\text {diffusion }}^{w}=\left(m_{w}, T\right) \\
& j^{M_{w}}=-K_{m_{w}}^{\vec{\Phi}_{\text {diffusion }}^{w}} \cdot \frac{\mathrm{d} m_{w}}{\mathrm{~d} x}-K_{T}^{\vec{\Phi}_{\text {diffusion }}^{w}} \cdot \frac{\mathrm{d} T}{\mathrm{~d} x}
\end{aligned}
$$

Taking the sum of the currents (21) and (22) leads to the total current in the pressure-driven model, cf. Sect. 1.2, Eq. 3, plus thermodiffusion:

$$
j^{M_{w+v}}=-K_{p_{c}}^{\vec{\Phi}_{\text {pressure }}^{w}} \cdot \frac{\mathrm{d} p_{c}}{\mathrm{~d} x}-K_{p_{v}}^{\vec{\Phi}_{\text {pressure }}^{v}} \cdot \frac{\mathrm{d} p_{v}}{\mathrm{~d} x}-\left(K_{T}^{\vec{\Phi}_{\text {pressure }}^{v}}+K_{T}^{\vec{\Phi}_{\text {pressure }}^{w}}\right) \cdot \frac{\mathrm{d} T}{\mathrm{~d} x} .
$$

The sum of the currents (21) and (23) leads to the total current in the liquid diffusion/vapour pressure model, cf. Sect. 1.2, Eq. 5, plus thermodiffusion:

$$
j^{M_{w+v}}=-K_{m_{w}}^{\vec{\Phi}_{\text {diffusion }}^{w}} \cdot \frac{\mathrm{d} m_{w}}{\mathrm{~d} x}-K_{p_{v}}^{\vec{\Phi}_{\text {pressure }}^{v}} \cdot \frac{\mathrm{d} p_{v}}{\mathrm{~d} x}-\left(K_{T}^{\vec{\Phi}_{\text {pressure }}^{v}}+K_{T}^{\vec{\Phi}_{\text {diffusion }}^{w}}\right) \cdot \frac{\mathrm{d} T}{\mathrm{~d} x}
$$

The models described by (24) and (25) are equivalent according to their derivation. They should even be equivalent to any other model derived from the state-variable approach with two independent state variables.

A fourth possibility is to choose moisture content and temperature $\left(m_{w+v}, T\right)$ as state variables and total moisture mass $M_{w+v}$ as transported quantity. According to (20) the moisture current reads:

$$
\begin{aligned}
& \vec{\Phi}_{\text {diffusion }}=\left(m_{w+v}, T\right) \\
& j^{M_{w+v}}=-K_{m_{w+v}}^{\vec{\Phi}_{\text {diffusion }}} \cdot \frac{\mathrm{d} m_{w+v}}{\mathrm{~d} x}-K_{T}^{\vec{\Phi}_{\text {diffusion }}} \cdot \frac{\mathrm{d} T}{\mathrm{~d} x}
\end{aligned}
$$

This equation corresponds to the diffusivity model, cf. Sect. 1.2, Eq. 6. 
There arises the question, why the model in (26) has two independent gradients and not three, as in (24) and (25). According to the general linear approach any model based on two state variables can have at most two independent gradients. The third gradient in the latter models stems from the fact, that the liquid and vapour currents were determined separately. However, in the assumption of two independent state variables, the evaporation equilibrium between water and vapour is assumed implicitly. This fact can be used to eliminate one of the three gradients. Choose as independent state variables $\left(m_{w}, T\right)$. Then an evaporation equilibrium must exist in the form $p_{v}\left(m_{w}, T\right)$. The vapour pressure gradient becomes

$$
\frac{\mathrm{d} p_{v}(x)}{\mathrm{d} x}=\frac{\partial p_{v}\left(m_{w}, T\right)}{\partial m_{w}} \frac{\mathrm{d} m_{w}}{\mathrm{~d} x}+\frac{\partial p_{v}\left(m_{w}, T\right)}{\partial T} \frac{\mathrm{d} T}{\mathrm{~d} x}
$$

Inserted into Eq. 25, the diffusion model (26) is obtained:

$$
\begin{aligned}
j^{M_{w+v}}= & -\underbrace{\left(K_{m_{w}}^{\vec{\Phi}_{\text {diffusion }}^{w}}+K_{p_{v}}^{\vec{\Phi}_{\text {pressure }}^{v}} \frac{\partial p_{v}\left(m_{w}, T\right)}{\partial m_{w}}\right)}_{K_{m_{w}}^{\vec{\Phi}_{\text {diffusion }}}} \cdot \frac{\mathrm{d} m_{w}}{\mathrm{~d} x} \\
& -\underbrace{\left(K_{T}^{\vec{\Phi}_{\text {pressure }}^{v}}+K_{T}^{\vec{\Phi}_{\text {diffusion }}^{w}}+K_{p_{v}}^{\vec{\Phi}_{\text {ressure }}^{v}} \frac{\partial p_{v}\left(m_{w}, T\right)}{\partial T}\right)}_{K_{T}^{\Phi_{\text {diffusion }}}} \cdot \frac{\mathrm{d} T}{\mathrm{~d} x}
\end{aligned}
$$

In analogy, the pressure-driven model (24) can be transformed using the evaporation equilibrium in the form $p_{c}\left(p_{v}, T\right)$. Then, the Glaser model including thermodiffusion is obtained:

$$
\begin{aligned}
j^{M_{w+v}}= & -\underbrace{\left(K_{p_{c}}^{\vec{\Phi}_{\text {pressure }}^{w}} \frac{\partial p_{c}\left(p_{v}, T\right)}{\partial p_{v}}+K_{p_{v}}^{\left.\vec{\Phi}_{\text {pressure }}^{v}\right)}\right)}_{K_{p_{v}}^{\Phi_{\text {Glaser }}}} \cdot \frac{\mathrm{d} p_{v}}{\mathrm{~d} x} \\
& -\underbrace{\left(K_{T}^{\vec{\Phi}_{\text {pressure }}^{v}}+K_{T}^{\vec{\Phi}_{\text {pressure }}^{w}}+K_{p v}^{\vec{\Phi}_{\text {pressure }}^{v}} \frac{\partial p_{v}\left(p_{c}, T\right)}{\partial T}\right)}_{K_{T}^{\vec{\Phi} \text { Glaser }}} \cdot \frac{\mathrm{d} T}{\mathrm{~d} x}
\end{aligned}
$$

As can be seen, all moisture current equations derived from the general linear approach are equivalent, if the transport coefficients are considered to be arbitrary phenomenological coefficients. All equations have two independent driving potentials and two independent conductivities to account for the same total moisture current $j^{M_{w+v}}$. However in the case of the phase-divided models there are two additional independent conductivities, which describe the relationship between liquid and vapour transport.

It is only model specific, how the currents are subdivided into partial currents, which are e.g. pressure-driven, moisture-content driven or temperature-driven. Even for the same gradient, (e.g. $\mathrm{d} T / \mathrm{d} x$ in case of the thermodiffusion) the partial currents may vary in different models, depending on the other variable (e.g. moisture content or liquid pressure). As can be calculated, the difference of liquid thermodiffusion becomes in the models (24) and (25):

$$
\begin{aligned}
j_{\text {diffusion }, \mathrm{T}}^{M_{w}}-j_{\text {pressure } \mathrm{T}}^{M_{w}} & =\left(K_{T}^{\vec{\Phi}_{\text {pressure }}^{w}}-K_{T}^{\vec{\Phi}_{\text {diffusion }}^{w}}\right) \cdot \frac{\mathrm{d} T}{\mathrm{~d} x} \\
& =K_{m_{w}}^{\vec{\Phi}_{\text {diffusion }}^{w}} \cdot \frac{\partial m_{w}\left(p_{c}, T\right)}{\partial T} \frac{\mathrm{d} T}{\mathrm{~d} x}
\end{aligned}
$$




\section{The Thermodynamic and the Micromechanical Approach}

The derivations of transport currents in the literature can be classified into two types: Either they are based on thermodynamics and investigate the entropy production, or they consider explicitly the transport processes on a microscopic scale. The former will be called the "thermodynamic approach", the latter the "micromechanical approach" throughout this publication.

The general linear approach gives no information about the order of magnitude of the different transport coefficients $K$. Especially there is no argument for the neglect of some of the independent transport processes (like thermodiffusion in the non-hysteretic heat and moisture transport).

\subsection{Derivation and Properties of the Thermodynamic Approach}

In the thermodynamic approach, the currents $j^{W}$ and their respective driving forces are obtained from the entropy production. The transport equation (8) for the entropy $S$ (De Groot and Mazur 1962) is given by:

$$
\frac{\partial}{\partial t} S(\vec{x}, t)=-\sum_{k} \frac{\partial}{\partial x_{k}} j_{k}^{S}(\vec{x}, t)+\sigma^{S}(\vec{x}, t) .
$$

The entropy current and the entropy content are stated as $\vec{j} S(\vec{x}, t)$ and $s(\vec{x}, t)$, respectively. The entropy production rate per volume is given by $\sigma^{S}(\vec{x}, t)$.

The elaborate derivation of $\sigma^{S}(\vec{x}, t)$ for a heat, air moisture and salt transport model including diffusion has been done by Grunewald $(1997,2000)$ and for a heat, air and liquid water transport model including surface effects by Hassanizadeh (1990) and Gray (1991). The derivations for the moisture flows are summarised in the appendix, sections A.1-A.2. Grunewald (2000) obtained the temperature-constant gradient of the chemical potential as the thermodynamic driving force for the diffusive water vapour flow. It can be shown that this corresponds to the vapour pressure gradient. Gray and Hassanizadeh obtained the force exerted by the solid-liquid interface on the liquid phase as the driving force of the liquid water flow. They showed that this force in turn could be identified with the liquid or capillary pressure gradient.

To derive the general pros and contras of the thermodynamic approach, it is necessary to investigate the derivation of this approach in more detail. A review is given by Jaynes (1980), whose results will be applied to our problem of spatial transport in a porous system. Important assumptions and properties of the system are in this case:

- The time-dependent local system state can be described by a full set of extensive conserved properties $\vec{w}$ (energy, masses, ...) in different regions of the REV, cf. Fig. 1. If some of these properties are in exchange equilibrium with other regions, (e.g. vapour mass and liquid water mass) only the total amount will be accounted for.

- Any change of the system state $\vec{w}$ corresponds to a flow, either inside the reference volume itself (e.g. due to a phase change) or to the surrounding (i.e. the transport currents).

- The entropy production of each independent transport process has to be greater or equal zero according to the second law of thermodynamics. Inside the REV all flows causing a change of $\vec{w}$ must be irreversible, according to the above definition. The transport currents over the system boundaries are considered as non-equilibrium flows (i.e. irreversible, not quasistatic, friction). Therefore, in the case considered here, the entropy production will be greater zero, if the system is not in equilibrium. 
- Assuming that flows inside the REV's do not interact with the spatial flows, cf. e.g. Grunewald (1997), and there are no interactions between spatial flow components in different directions $(x, y, z)$, it is sufficient to consider only spatial flows in a 1-dimensional system.

- To derive the transport currents, the system can be considered as closed containing two identical neighbouring volume elements cf. Fig. 2. A set of independent state variables of the combined system is given by the average and the difference of $\vec{W}_{1}, \vec{W}_{2}$ :

$$
\begin{aligned}
\vec{W} & \equiv 1 / 2 \cdot\left(W_{1}+W_{2}\right), \\
\Delta \vec{W} & \equiv 1 / 2 \cdot\left(W_{2}-W_{1}\right)-\Delta W_{0}\left(\Delta \vec{Y}_{\text {mat }}\right),
\end{aligned}
$$

where the time-independent quantity $\Delta \vec{W}_{0}\left(\Delta \vec{Y}_{\text {mat }}\right)$ is chosen in such a manner, that in the equilibrium state of the system $\Delta \vec{W}$ becomes zero, even if there are differences $\Delta Y_{\text {mat }, i}$ in the material properties of the two volumes. According to conservation laws, $\vec{W}$ is a conserved parameter, whereas $\Delta \vec{W}$ is not. The current through the common boundary of the volumes becomes

$$
j^{\vec{W}}=\mathrm{d} / \mathrm{d} t(\Delta \vec{W}) .
$$

From this point, the description of Jaynes (1980) can be adapted.

The state of a general closed system is assumed to be near equilibrium. The deviations hereof are described by non-conserved parameters $a_{i}$. In the above-described case, these deviations are given by the differences $\Delta \vec{W}_{i}$ :

$$
\Delta \vec{W}_{i} \stackrel{\wedge}{=} \vec{a}_{i}
$$

Then the entropy can be Taylor-expanded (Jaynes 1980), Eq. 4:

$$
\begin{aligned}
S & =S_{0}-(1 / 2) \Sigma_{i j} G_{i j} \cdot a_{i} \cdot a_{j} \\
G_{i j} & \equiv-\partial^{2} S / \partial a_{i} \partial a_{j}
\end{aligned}
$$

$S_{0}$ is the maximum of the entropy function $S(\vec{a})$ and $G_{i j}$ is a symmetric matrix, which describes the near equilibrium behaviour of the system. $S$ has an absolute maximum for $\vec{a}=0$. Therefore, $\tilde{G}$ must be positive definite, i.e. for arbitrary $\vec{a} \neq 0$ it holds

$$
\vec{a}^{T} \cdot \tilde{G} \cdot \vec{a}>0 .
$$

The entropy production due to a time-dependent change of the system can be written in the well-known form:

$$
\mathrm{d} S / \mathrm{d} t=\Sigma_{i} \partial S / \partial a_{i} \cdot \mathrm{d} a_{i} / \mathrm{d} t=\Sigma_{i} F_{i} \cdot J^{W_{i}}
$$

where $F_{i}$ are defined by

$$
F_{i} \equiv \partial S / \partial a_{i}=-\Sigma_{j} G_{i j} a_{j}
$$

Because the $F_{i}$ give by definition the entropy production (37), they represent as "driving forces" the tendency of the system to reach equilibrium via the currents $J^{W_{i}}$ in the thermodynamic approach.

These forces can also be related to the arbitrary gradients of the general linear approach: If the state variables and material properties of the two volume elements are equal, the system 
is in equilibrium and the forces $F_{i}$ must vanish. Taylor expansion of the forces with respect to the gradients $\vec{\nabla} \vec{Y}, \vec{\nabla} \vec{Y}_{\text {mat }}$ of state variables $\vec{Y}$ and material properties $\vec{Y}_{\text {mat }}$, cf. (13), yields

$$
\begin{aligned}
F_{i}\left(\vec{Y}, \vec{Y}_{\text {mat }}, \vec{\nabla} \vec{Y}, \vec{\nabla} \vec{Y}_{\text {mat }}\right) & =\Sigma_{j} \partial F_{i} / \partial \vec{\nabla} Y_{j} \cdot \vec{\nabla} Y_{j}+\Sigma_{j^{\prime}} \partial F_{i} / \partial \vec{\nabla} Y_{\text {mat }, j^{\prime}} \cdot \vec{\nabla} Y_{\text {mat }, j^{\prime}} \\
& \equiv \Sigma_{j, j^{\prime}} A_{i j} \cdot \vec{\nabla} Y_{j}+A_{i j^{\prime}} \cdot \vec{\nabla} Y_{\text {mat }, j^{\prime}}
\end{aligned}
$$

This is the general relationship between the thermodynamic approach and the general linear approach. The thermodynamic driving forces can be written as linear combinations of state variable gradients.

However, the forces can also be identified by the gradient, of a thermodynamic potential $\Phi_{i}$, defined by

$$
F_{i} \stackrel{!}{=}-\vec{\nabla} \Phi_{i}
$$

From definition (40) and from

$$
\vec{\nabla} \Phi_{i}\left(\vec{Y}, \vec{Y}_{\text {mat }}\right)=\Sigma_{j} \partial \Phi_{i} / \partial Y_{j} \cdot \vec{\nabla} Y_{j}+\Sigma_{j^{\prime}} \partial \Phi_{i} / \partial Y_{\text {mat }, j^{\prime}} \cdot \vec{\nabla} Y_{\text {mat }, j^{\prime}}
$$

it follows

$$
\partial \Phi_{i} / \partial Y_{j}=-A_{i j}
$$

Therefore, the potential $\Phi(\vec{Y})$ can be obtained via integration over the $A_{i j}$, provided the coefficients $A_{i j}$ are integrable.

If every spontaneous change of the system is due to an entropy increase, then from (37) follows:

$$
j^{W}(\vec{F}=0)=0
$$

This corresponds to Eq. 16 of the general linear approach. In the case here, the currents vanish for zero driving forces according to the entropy production principle. In the general linear approach, Eq. 16, the currents vanished for zero state variable gradients according to the symmetry principle. In the same manner as in the general linear approach, Eq. 17, the currents can be derived via linear Taylor expansion, cf. also (Callen 1985), chapter 14-3:

$$
j^{W_{i}}(\vec{F})=\frac{\mathrm{d} a_{i}}{\mathrm{~d} t}(\vec{F})=\Sigma_{j} \partial j^{W_{i}}(\vec{F}) / \partial F_{j} \cdot F_{j} \equiv \Sigma_{j} L_{i j} F_{j}
$$

If the coefficients $L_{i j}$ are identified with the conductivities $K_{i j}$ and the forces $F_{j}$ with the state variable gradients $\vec{\nabla} Y_{i}$, this equation corresponds to (20) in the general linear approach.

Now the question is, whether additional statements can be made for the $L_{i j}$, as compared to the $K_{i j}$ due to the entropy production principle. First, as compared to (20), the space dependent material property gradients $\vec{\nabla} Y_{\text {mat }}$ have disappeared. Second, the famous Onsager theorem (Jaynes 1980), Eq. 14, follows from the reversibility of microscopic system changes:

$$
\tilde{L}=\tilde{L}^{T} \quad \text { or } \quad L_{i j}=L_{j i}
$$

By this the number of off-diagonal transport coefficients is reduced. Third, the above relationships $\mathrm{d} S / \mathrm{d} t=\Sigma_{i} J_{i} \cdot F_{i}$ and $J_{i}=\Sigma_{j} L_{i j} F_{j}$ suggest, that the main contribution of the entropy production due to the current $J_{i}$ stems from the force $F_{i}$ (and not from forces $F_{j \neq i}$ ). Therefore the current $J_{i}$ should be mainly due to the force $F_{j}$ and therefore the diagonal 
elements of the matrix $L_{i j}$ should be larger than the off-diagonal ones. More precisely, the combination of (37) and (44) gives

$$
\mathrm{d} S / \mathrm{d} t=\vec{F}^{T} \tilde{L} \vec{F} \geq 0
$$

Since the entropy production must be greater or equal zero for arbitrary forces $\vec{F}$, Eq. 46 states, that the matrix $\tilde{L}$ is positive semi-definite. However, this does not lead to the desired conclusion, that the matrix $\tilde{L}$ is diagonally dominant. The relationship would hold only in the inverse direction: If $\tilde{L}$ is strong diagonally dominant, i.e. $\left|L_{i i}\right|>\Sigma_{j \neq i}\left|L_{i j}\right|$ and all diagonal entries are greater zero, then $\tilde{L}$ is also positive definite.

As $\tilde{G}$ and $\tilde{L}$ are both symmetric or self-adjoint, they can be orthogonally transformed by a matrices $\tilde{O}_{L}, \tilde{O}_{G}$ into diagonal forms $\tilde{L}_{D}, \tilde{G}_{D}$ (Jaynes 1980), p. 588. Additionally, because $\tilde{G}$ and $\tilde{L}$ are positive (semi)definite, Eqs. 36 and 46 , the values of $\tilde{G}_{D}$ are greater zero and the values of $\tilde{L}_{D}$ are greater or equal zero. This leads to a reformulation of (44):

$$
\begin{aligned}
\vec{J} & =\tilde{L} \cdot \vec{F} \\
\tilde{O}_{L} \cdot \vec{J} & =\tilde{O}_{L} \cdot \tilde{L} \cdot \tilde{O}_{L}^{-1} \cdot \tilde{O}_{L} \cdot \vec{F} \\
\vec{J}^{\prime} & =\tilde{L}_{D} \cdot \vec{F}^{\prime}
\end{aligned}
$$

Therefore, in an appropriately chosen system, the coefficient matrix $\tilde{L}$ becomes diagonal. The choice of the system corresponds to the choice of the transported currents, i.e. to the choice of $\vec{J}^{\prime}$, from which the driving forces $\vec{F}^{\prime}$ can be derived and afterwards the coefficient matrix $\tilde{L}$ becomes diagonal. Unfortunately, it is necessary to know the full matrix $\tilde{L}$ in advance. Then the orthogonal transformation $\vec{O}_{L}$ can be determined and finally the system of currents $\vec{J}^{\prime}=\tilde{O}_{L} \cdot \vec{J}$ can be determined.

This would, once more, require the determination of the non-diagonal coefficients by microphysical modelling or by experiment. Therefore, it is suggested (Jaynes 1980) p. 588, that the transformation $\tilde{O}_{L}$ could be identical with the transformation $\tilde{O}_{G}$ which makes the matrix $\tilde{G}$ diagonal. In case of a diagonal $\tilde{G}_{D}$, all corresponding deviations $a_{i}^{\prime}$ from equilibrium lead independently to an entropy increase, since from (35) the deviation of $S$ from the equilibrium $S_{0}$ is

$$
S-S_{0}=-\Sigma_{i} G_{i i}^{D} \cdot\left(a_{i}{ }^{\prime}\right)^{2} .
$$

There are no mixed terms containing $a_{i}{ }^{\prime} \cdot a_{j}{ }^{\prime}$ in (48). The determination of $\tilde{G}$ can be done, if from the thermodynamic model the entropy of a volume element containing a gradient

$$
S(\vec{a})=S(\Delta \vec{W})=S(\operatorname{grad} \vec{w})
$$

is known. However, according to the theorem of simultaneous diagonalisability (Jaynes 1980), p. 588, the diagonal form for $\tilde{G}$ and $\tilde{L}$ is only achieved by the same transformation $O$, if the additional requirement is made, that $\tilde{L}$ and $\tilde{G}$ commute, i.e.

$$
\tilde{L} \cdot \tilde{G}=\tilde{G} \cdot \tilde{L}
$$

Jaynes suggests, that this theorem should be checked for systems in which $L$ and $G$ are determined independently.

The independent determination of the non-diagonal coefficients of $\tilde{L}$ requires an experiment or a numerical simulation. According to the Prigogine theorem, described in (Jaynes 1980), p. 581, if a potential difference (e.g. Temperature) is applied at the boundary of a system, then the system turns into a stationary state at which the entropy production is minimal. If one gradient is given fixed over the boundary and the other gradients are free, then the latter 
will take a form by which the entropy production is minimal. The description of Jaynes will be demonstrated now for the case of non-hysteretic heat and moisture transport. The latent heat current $J_{Q}$ and the particle current (Moisture current) $J_{N}$ are chosen as independent currents $W_{i}$. An experimental setup corresponding to this situation would be a homogeneous volume filled with particles and with impermeable walls to which a temperature gradient is applied. The particle concentration gradient (i.e. the gradient of the chemical potential) can freely respond to the given conditions.

$$
\begin{aligned}
\vec{J} & =\left(\begin{array}{c}
\vec{j}^{Q} \\
\vec{j}^{N}
\end{array}\right) \Rightarrow \vec{F} \equiv\left(\begin{array}{c}
\vec{F}^{Q} \\
\vec{F}^{N}
\end{array}\right)=\left(\begin{array}{l}
\operatorname{grad}(1 / T) \\
\operatorname{grad}(\mu)_{T}
\end{array}\right) \\
\sigma^{S} & =\tilde{L} \cdot \vec{F}=L_{Q Q} F_{Q}{ }^{2}+2 L_{Q N} F_{Q} F_{N}+L_{N N} F_{N}^{2} \\
\partial \sigma^{S} / \partial F_{N} & =2 L_{N N} F_{N}+2 L_{Q N} F_{Q} \stackrel{!}{=} 0 \text { (Prigogine) } \\
& \Rightarrow J_{N}=\Sigma_{j} L_{N j} F_{j}=L_{N N} F_{N}+L_{N Q} F_{Q}=\partial \sigma^{S} / \partial F_{N}=0
\end{aligned}
$$

In this case, the Prigogine theorem yields just the vanishing particle current $J_{N}$. The size of non-diagonal effect can now be determined because the thermodiffusion coefficient $L_{N Q}$ must obey the relation

$$
L_{N Q} / L_{N N}=-F_{N} / F_{Q}
$$

Thus, from the gradients of the chemical potential $F_{N}$ the size of the thermodiffusion $L_{N Q}$ can be determined.

To summarise, in the thermodynamic approach there is no dependency of the transport processes on material property gradients (other than in the general linear approach). The diagonal conductivity coefficients $L_{i i}$ can be estimated more or less larger than the non-diagonal ones, due to the positive definiteness of $\tilde{L}$. If an appropriate choice of the transport currents $\vec{J}$ is made, the non-diagonal coefficients can be eliminated. However, this would still require a complete determination of all coefficients $L_{i j}$. According to Jaynes (1980), the optimum choice of transport currents could also be estimated from the explicit expression for $S(\vec{\nabla} \vec{w})$ from which the matrix $\tilde{G}$ can be determined according to (35). He considers this as a central point of the thermodynamic model (p. 589): “...the relation $L G=G L$. If his conjecture should be confirmed, irreversible thermodynamics would become more useful..."

\subsection{Derivation and Properties of the Micromechanical Approach}

In principle, the heat and mass transport processes can also be calculated on the microscopic scale. This requires a more detailed knowledge of the physical and chemical properties of the transported masses, of the pore configuration and the properties of the pore surfaces. The main feature of the micromechanical approach is that it provides explicit expressions for the transport coefficients $K$. In the general case, a micromechanical modelling of the transport currents can be done via simplified analytical models or via numerical investigations such as Monte Carlo simulation, molecular dynamical simulation etc.

For the example of the non-hysteretic heat and moisture transport an analytic micromechanical investigation leads to the following results: The water vapour flow is obtained from the theory of ideal gas mixtures (Bednar 2000). The corresponding driving potential is the vapour pressure, however, with a small contribution of thermodiffusion, cf. Appendix A.3. The liquid water flow and the liquid conductivity are obtained from the Navier-Stokes equations in the pore system (Whitaker 1986a), cf. Appendix A.4. The corresponding driving potential is the liquid pressure. The effect of thermodiffusion can be considered negligible 
under the assumption of temperature-independent porosity, liquid water density, viscosity and surface tension:

$$
\Phi_{\text {por }}=\text { const } ; \quad \rho_{\ell}=\text { const; } \quad \eta_{\ell}=\text { const } ; \quad \gamma_{\ell g}=\text { const }
$$

\subsection{Application to the Example Models}

Application to the pressure-driven model: The pressure-driven model including thermodiffusion had been obtained from the general linear approach, cf. Eq. 24. The driving potentials for the phase separated transport were liquid pressure, water vapour pressure and temperature. The thermodynamic and the micromechanical approach stated for this case a comparatively small thermodiffusion:

$$
\left\{\begin{array}{l}
\text { thermodynamic approach } \\
\text { micromechanical approach }
\end{array}\right\} \Rightarrow K_{T}^{\vec{\Phi}_{\text {pressure }}^{v}} \approx 0 \text { and } K_{T}^{\vec{\Phi}_{\text {pressure }}^{w}} \approx 0
$$

This yields the pressure-driven model without thermodiffusion, Eq. 3, which could not be obtained from the general linear approach alone.

Application on the liquid diffusivity/vapour pressure model: The liquid diffusivity/vapour pressure model plus thermodiffusion had also been obtained from the general linear approach, cf. Eq. 25. In this case the potentials were liquid water content, water vapour pressure and temperature. A vanishing thermodiffusion can only be deduced from the pressure-driven model. According to (30), this requires a temperature independent moisture retention:

$$
\partial m_{w}\left(p_{c}, T\right) / \partial T=0 .
$$

Equation (54) is an additional assumption. It holds for the simple well-known capillary condensation theory stating that with increasing moisture content water fills the smallest pores first and then the larger ones. The capillary pressure becomes a function of the water content, of the pore size distribution (the porosity) and the pore shape of the material and of the liquid water surface tension and density. With the exception of the water content, all other quantities are assumed to be invariant liquid or material properties. If all these quantities are nearly temperature-independent as has also been assumed in the micromechanical approach, Eq. 52, the moisture content becomes a function of capillary pressure alone:

$$
m_{w}\left(p_{c}, f_{\text {pore shape }}, \gamma_{\ell g}, \rho_{\ell}, f_{\text {pore size }}\right)=m_{w}\left(p_{c}\right)
$$

This is consistent with Eq. 54.

According to Rouquerol et al. (1999) the theory of capillary condensation is fully valid only in the mesopore region $2 \mathrm{~nm}<r_{\max }<50 \mathrm{~nm}$. At very small and at very large pores and in organic materials, the moisture retention may become temperature dependent.

To summarize, from the thermodynamic and the micromechanical approaches it follows, that the effect of thermodiffusion can be neglected in the pressure-driven model. For all other models, the thermodiffusion must be deduced from the pressure-driven model. For the liquid diffusivity / vapour pressure model the neglect of thermodiffusion can only be done, if the moisture retention in the form $m_{w}\left(p_{c}, T\right)$ turns out to be independent of temperature.

Note, that the pressure-driven model has still two independent gradients, despite of the neglect of thermodiffusion. Therefore, it can be derived from the general linear approach alone. However, the neglect of the thermodiffusion in each phase (liquid and vapour) leads to the additional information, that if the driving potentials $p_{v}, p_{c}$ are chosen, these driving potentials account for the vapour and liquid moisture transport separately. Also, the explicit expressions for the corresponding vapour and liquid conductivity coefficients are obtained, 
cf. Appendix A.3 and A.4. The micromechanical model can be improved, if the interaction of the vapour and the liquid transport is considered (Scheffler et al. 2005).

\section{The Three Approaches Applied to More Complex Models}

The same general considerations made for the non-hysteretic heat and moisture transport apply to other systems with two state variables as well (e.g. isothermal moisture and air transport). However, if the number of state variables increases linearly, the number of independent transport processes increases quadratically. Consider the case of heat and moisture transport with an additional conserved quantity, such as air or salt or a hysteretic water content. If this quantity is denoted by $B$, then an additional state variable $Y_{B}$ will be needed to describe the system and three independent currents $j^{Q}, j^{M_{w+v}}, j^{B}$ arise (heat, moisture and $B$-current). If the arbitrary independent state variables are denoted $\vec{Y}=\left(Y_{1}, Y_{2}, Y_{B}\right)$ then the general linear approach leads to the equations for the independent currents according to (20):

$$
\left(\begin{array}{l}
j^{Q} \\
j^{M_{w+v}} \\
j^{B}
\end{array}\right)=\left(\begin{array}{lll}
K_{11}^{\vec{Y}} & K_{12}^{\vec{Y}} & K_{1 B}^{\vec{Y}} \\
K_{21}^{\vec{Y}} & K_{22}^{\vec{Y}} & K_{2 B}^{\vec{Y}} \\
K_{B 1}^{\vec{Y}} & K_{B 2}^{\vec{Y}} & K_{B B}^{\vec{Y}}
\end{array}\right) \cdot\left(\begin{array}{l}
\operatorname{grad} Y_{1} \\
\operatorname{grad} Y_{2} \\
\operatorname{grad} Y_{B}
\end{array}\right)
$$

The conductivity coefficients $K_{11}$ and $K_{22}$ denote the heat and moisture conductivity, the coefficients $K_{21}$ and $K_{12}$ denote the Dufour effect and the Soret effect (thermodiffusion). In addition, there are five independent transport coefficients describing the transport of quantity $B$. To reduce the number of coefficients, the thermodynamic or the micromechanical approach have to be employed. If the thermodynamic approach is used, the vector of the three thermodynamic potentials corresponding to the three given independent currents has to be used as vector of state variables: $\vec{Y} \equiv \vec{\Phi}^{T D}$. Then from the Onsager-Relations the $K$-Matrix becomes symmetric:

$$
K_{i j}^{\vec{\Phi}^{T D}}=K_{j i}^{\vec{\Phi}^{T D}}
$$

Thus, the number of independent transport coefficients for the whole system reduces to 6 . If the non-diagonal coefficients could be treated as small (as discussed above), then the coefficients $K_{21}, K_{B 1}$ and $K_{B 2}$ could be neglected.

\section{Summary and Conclusions}

In this work three different derivation schemes for spatial transport currents have been compared in a systematic way. First, a general linear approach has been formally derived from a symmetry principle. It turned out, that each model using a complete set of independent state variable gradients with their associated conductivities is able to describe in a linear approximation all transport processes in a homogeneous material, if the corresponding transport coefficients are "phenomenological", i.e. arbitrary functions of the given state variables. Then the thermodynamic and the micromechanical approach often encountered in literature have been investigated. The thermodynamic approach selects from the arbitrary set of state variables a set of thermodynamic potentials corresponding to a set of independent transport currents. If these potentials are used, the dependency on the material property gradients, causing problems in inhomogeneous materials and at material boundaries disappears. Also, the 
independent transport coefficient matrix $\tilde{L}=\widetilde{K}\left(\vec{\Phi}^{T D}\right)$ contains less independent transport coefficients due to the well-known symmetry given by the Onsager relations. The non-diagonal coefficients are only in tendency smaller than the diagonal ones, due to the positive definiteness of $\widetilde{L}$, which follows from the principle of positive entropy production. There is always an optimum choice for the transport currents $J$, for which the non-diagonal coefficients vanish, but as a rule the non-diagonal coefficients are non-zero.

Finally, the micromechanical approach gives the most detailed insight into the transport processes. The transport processes are derived from the microscopic physical laws and the order of magnitude of their transport coefficients can be estimated.

The above-considerations have been applied to the simple case of non-hysteretic heat and moisture transport. The different expressions for the moisture transport currents, which are known from literature, can be derived to a large part by the general linear approach. Sometimes a term accounting for thermodiffusion must be added. As long as two independent gradients are employed, all expressions depending on two independent driving potentials are equivalent, if the transport coefficients are considered to be arbitrary functions. It is well-known, that the driving potentials of moisture transport are the liquid and vapour pressure. These potentials are obtained from the thermodynamic and micromechanical approach. If these driving potentials are used, then the dependency of the material property gradients is eliminated. Moreover, it can be expected from the thermodynamical approach, that the thermodiffusion in the liquid and vapour phase becomes small for each phase in the pressure-driven model. This can definitively be confirmed by the micromechanical approach. However, since the pressure-driven model still contains two independent gradients, it could have been derived by the general linear approach alone. The neglect of thermodiffusion results in the additional statement, that the vapour and liquid pressure are driving potentials for the vapour and liquid transport separately. Also, different micromechanical models for the liquid and vapour transport coefficients have been cited from literature.

For systems containing more than two state variables the number of independent transport processes and coefficients increases rapidly; therefore the thermodynamic or the micromechanical approach have to be applied to reduce the number of transport coefficients. The general linear approach however, gives immediately the maximum number of independent transport processes, if no simplifications (except local equilibrium assumptions and linearity) are made.

\section{Appendix}

\section{A.1 Thermodynamic Approach for the Vapour Transport According to Grunewald}

Grunewald (2000), Eq. 1.43 obtains the following expression for the entropy production according to (31) for a system of separate phases $\alpha=\{$ liquid, solid, gaseous $\}$, each consisting of several components $i$ (dry air and water vapour, liquid water and dissolved salt):

$$
\begin{aligned}
\sigma^{S_{\alpha}} \cdot T_{\alpha}= & -\frac{1}{T_{\alpha}} \cdot j_{k}^{Q} \cdot \frac{\partial T_{\alpha}}{\partial x_{k}}-\Sigma_{i} j_{k, \text { diff }}^{M_{\alpha i}} \cdot\left[\left(\frac{\partial \mu_{i}}{\partial x_{k}}\right)_{T_{\alpha}}-g_{k}\right] \\
& -\pi_{j k, \alpha} \cdot \frac{\partial v_{j}^{M_{\alpha}}}{\partial x_{k}}+\sigma_{\text {scalar }}^{S_{\alpha}} \cdot T_{\alpha}
\end{aligned}
$$


The four summands contain the following transport currents: $j_{k}^{Q}$ is the heat conduction flow, $j_{k \text {,diff }}^{M_{\alpha i}}$ are the diffusion flows (water vapour diffusion $i=v$ or the salt diffusion $i=s$ ). The pressure tensor $\pi_{j k, \alpha}$ represents the momentum flow and the last term containing $\sigma_{\text {scalar }}^{S_{\alpha}}$ is due to phase change flows and heat inflow from external sources.

The driving forces are the following: The vapour mass diffusion is driven by the chemical potential gradient in the gaseous phase $\alpha=g$ at constant temperature, denoted by $\left(\partial \mu_{v} / \partial x\right)_{T}$. The gravity force $g_{k}$ will be neglected hereafter. The chemical potential is assumed to depend on temperature, total gas pressure $p_{g}$ and water vapour mass concentration $c_{v} \equiv M_{v} /\left(M_{a}+M_{v}\right)$. This leads to the following precise definition:

$$
\left(\frac{\partial \mu_{v}\left(p_{g}, c_{v}, T\right)}{\partial x_{k}}\right)_{T} \equiv \frac{\partial \mu_{v}}{\partial x_{k}}-\frac{\partial \mu_{v}}{\partial T} \frac{\partial T}{\partial x_{k}}=\frac{\partial \mu_{v}}{\partial p_{g}} \frac{\partial p_{g}}{\partial x_{k}}+\frac{\partial \mu_{v}}{\partial c_{v}} \frac{\partial c_{v}}{\partial x_{k}}
$$

Additionally it has to be considered that (58) gives two independent diffusive currents for dry air and for vapour transport, respectively. In fact, these two currents are not independent, but equal and of opposite sign so that the diffusion result into a zero net current. Taking the vapour diffusion current as independent and the air diffusion current as dependent, the entropy production due to vapour diffusion arises from the two currents together. This can be calculated from (58):

$$
\sigma_{\text {diffusion }}^{S}=-j_{k, \text { diff }}^{M_{v}} \cdot \frac{1}{T} \cdot\left[\left(\frac{\partial \mu_{v}}{\partial x_{k}}\right)_{T}-\left(\frac{\partial \mu_{a}}{\partial x_{k}}\right)_{T}\right]
$$

According to Callen (1985), Eq. 13.8, the chemical potential of the water vapour component in an ideal gas consisting of dry air and water vapour is given by:

$$
\mu_{v}\left(p_{g}, c_{v}, T\right)=R T\left(f_{v}(T)+\ln \left(p_{g}\right)+\ln \left(c_{v}\right)\right)
$$

where $p_{g}$ is the total gas pressure, $R$ is the universal gas constant, $c_{v}$ the vapour mass or particle concentration and $f_{v}(T)$ is a function depending only on temperature. An analogous relationship holds for the chemical potential of dry air $\mu_{a}\left(p_{g}, c_{a}, T\right)$. Inserting (61) into (60) yields after some calculation:

$$
\sigma_{\text {vapour diffusion }}^{S}=-j_{k, \text { diff }}^{M_{v}} \cdot \frac{R}{p_{v}} \cdot \frac{\partial p_{v}}{\partial x_{k}}
$$

Thus, in the case of water vapour the gradient of the chemical potential (60) is proportional to the vapour pressure gradient, i.e. the vapour pressure gradient is the driving potential of the water vapour current.

\section{A.2 Thermodynamic Approach for the Liquid Transport According to Gray and Hassanizadeh}

Hassanizadeh and Gray $(1990,1991)$ obtain the driving potentials taking the phase and interface interactions into account. After making all simplifications to get at the non-hysteretic heat, air and liquid water flow, the expression for the entropy production becomes (Hassanizadeh 1990), Eq. 71:

$$
\begin{aligned}
T \cdot \sigma^{S}= & \sum_{k}\left(-\vec{v}_{k}^{M_{w}} \cdot \hat{\tau}_{k}^{\ell}-\vec{v}_{k}^{M_{g}} \cdot \hat{\tau}_{k}^{g}-\Sigma_{\alpha \beta} \vec{v}_{k}^{M_{\alpha \beta}} \cdot \hat{\tau}_{k}^{\alpha \beta}\right) \\
& +\sum_{k}\left(j_{k}^{Q} \cdot \frac{1}{T} \cdot \operatorname{grad}(T)\right)-\Phi_{\mathrm{por}} \cdot \partial_{t} s^{w} \cdot\left(p_{g}-p_{\ell}-p_{c}\right)
\end{aligned}
$$


The driving potentials of the liquid water flow and the gaseous air flow are $\hat{\tau}^{\ell}$ and $\hat{\tau}^{g}$, respectively. The driving potential for the heat flow $\vec{j} Q$ is the temperature $\operatorname{gradient} \operatorname{grad}(T)$. Additionally, there is a third term in the entropy production, which becomes non-zero in case of pressure non-equilibrium between liquid and gaseous phases. The driving potential for this equilibrium is the pressure difference between capillary, gaseous and liquid pressures $p_{c}, p_{\ell}, p_{g}$. The associated "flow", i.e. the system state change expressed in extensive variables, is the change in water saturation $s_{w}=V_{\ell} / V_{\ell+g}$.

The expression for $\hat{\tau}^{\ell}$ can be taken from the following formula for the production of entropy due to liquid water flow according to (Hassanizadeh 1990), Eq. 58:

$$
\begin{aligned}
\sigma_{\text {liquid flow }}^{S} & =\sum_{k} v_{k}^{M_{w}} \cdot \frac{1}{T} \cdot\left[p_{\ell} \cdot \frac{\partial \theta_{\ell}}{\partial x_{k}}-\rho_{\ell} \theta_{\ell} \cdot \frac{\partial A_{\ell}}{\partial s_{w}} \frac{\partial s_{w}}{\partial x_{k}}-\hat{T}_{\ell m}^{\ell}-\hat{T}_{\ell g}^{\ell}\right] \\
& \equiv-v_{k}^{M_{w}} \cdot \frac{1}{T} \cdot \hat{\tau}_{k}^{\ell}
\end{aligned}
$$

$T$ is the temperature of the REV, $p_{\ell}, \theta_{\ell}, \rho_{\ell}$ are pressure, volumetric content and density of the liquid water phase, respectively. $s_{w}$ is the water saturation and $A_{\ell}$ the free energy density.

The most important term of (64) is the momentum transfer, i.e. the force exerted from the solid material $(m)$ on the liquid phase $(l)$. It is denoted by $\hat{T}_{\ell m}^{\ell}$. In other words, if the liquid flows with a velocity $v_{k}^{M_{w}}$, mechanical work is done against the friction force of the pore walls, which is dissipated into heat (entropy production).

Hassanizadeh (1990) argues that at very low currents $v_{k}^{M_{w}}$ the term in brackets in (64) vanishes. This is because there is force equilibrium between the liquid and the pore walls. For very slow velocities, i.e. quasistatic processes, no entropy is produced. Therefore the force $\hat{\tau}^{\ell}$ can be interpreted as the non-equilibrium part of $\hat{T}_{\ell m}^{\ell}+\hat{T}_{\ell g}^{\ell}$. Because the non-equilibrium (=friction) forces arise only at non-zero velocities of liquid and gaseous phases, a linear approach can be made for $\hat{\tau}$ (Hassanizadeh 1990), Eq. 74:

$$
\hat{\tau}_{\ell}=R_{m}^{\ell} \cdot v_{k}^{M_{w}}+R_{g}^{\ell} \cdot v_{k}^{M_{g}} \approx R_{m}^{\ell} \cdot v_{k}^{M_{w}}
$$

Using the momentum transport equation one obtains (Gray 1991), Eq. 36:

$$
-\frac{\partial p_{c}}{\partial x_{k}}-\rho_{\ell} \cdot g_{k}+\rho_{\ell} \frac{\partial A_{\ell}}{\partial s_{w}} \cdot \frac{\partial s_{w}}{\partial x_{k}}=-R_{m}^{\ell} \cdot v_{k}^{M_{w}}=-\hat{\tau}
$$

Provided, that the so-called "wettability potential” $\partial A_{\ell} / \partial s_{w}$ in (66) is neglected, Hassanizadeh and Gray obtain the capillary pressure together with the gravity force $g_{k}$ as driving potential for the liquid moisture flow.

\section{A.3 Micromechanical Approach for the Vapour Transport According to Bednar}

The standard micromechanical approach based on scattering of an ideal gas (Gerthsen 2006), Sect. 5.2.6, is for the given system described by Bednar (2000), p. 43. It is assumed, that the vapour molecules scatter with the air molecules. For free air, i.e. outside the material, the total water vapour current is given as follows (Bednar 2000) Eq. 3.18:

$$
j_{\text {free air }, k}^{M_{v}}=-M_{\text {molecule }, v} \cdot \frac{1}{3} \ell \cdot \frac{\partial}{\partial x_{k}} \cdot\left(v_{\text {molecule }, v} \cdot n_{v}\right)
$$

In this equation, $M_{\text {molecule, } v}$ is the mass, $\ell$ is the mean free path, $v_{\text {molecule, } v}$ is the thermal velocity and $n_{\text {molecule, } v}$ is the density of the water vapour molecule. 
Using the ideal gas law, the Maxwell velocity distribution and the scattering theory with a molecular cross section $\sigma_{\text {molecule }}$, the vapour current in free air (without porous medium) can be obtained:

$$
\begin{aligned}
j_{\text {free air }, k}^{M_{v}} & =-\frac{2}{3 \sqrt{\pi}} \cdot \frac{\sqrt{M_{\text {molecule }, v} \cdot k_{B} \cdot T}}{p_{g} \cdot \sigma} \cdot \sqrt{T} \cdot \frac{\partial}{\partial x_{k}}\left(\frac{p_{v}}{\sqrt{T}}\right) \\
& \equiv-\delta_{v, \text { air }} \sqrt{T} \frac{\partial}{\partial x_{k}} \cdot\left(\frac{p_{v}}{\sqrt{T}}\right)
\end{aligned}
$$

For the case of constant gaseous pressure $p_{g}$, Bednar cites the "Stefan correction factor" $1 /\left(1-p_{v} / p_{g}\right)$. Finally, inside the porous medium, the vapour current is decreased due to several reasons as compared to free air: The free space for vapour diffusion in the porous medium is reduced. The currents cannot go straight, but they have to follow the direction of the pore tubes. The microscopic gradients will be different from the average currents of the REV due to liquid water shortcuts in the pores.

These effects are included into a resistance factor $\mu\left(m_{w}\right)$ depending on the given material and on the water content. The microscopic water vapour transport is then given by

$$
\begin{aligned}
j_{k}^{M_{v}} & =\underbrace{-\frac{\delta_{v, \text { air }}}{\mu\left(m_{w}\right)} \cdot \frac{1}{1-p_{v} / p_{g}}}_{\delta_{v}} \sqrt{T} \frac{\partial}{\partial x_{k}} \cdot\left(\frac{p_{v}}{\sqrt{T}}\right) \\
& =-\delta_{v} \cdot \frac{\partial p_{v}}{\partial x_{k}}+\delta_{v} \cdot \frac{p_{v}}{2 T} \cdot \frac{\partial T}{\partial x_{k}}
\end{aligned}
$$

Thus, the microscopic theory yields the driving potentials and the transport coefficients of the vapour transport. Additionally, the relation between thermodiffusion and the normal pressure-driven diffusion can be determined from (69).

$$
\frac{\text { thermodiffusion }}{\text { pressure driven diffusion }}=\frac{p_{v}}{2 T} \cdot \frac{\partial T / \partial x_{k}}{\partial p_{v} / \partial x_{k}}=\frac{1}{2} \cdot \frac{\delta T / T}{\delta p_{v} / p_{v}}
$$

$T$ varies between $273 \mathrm{~K}$ and $300 \mathrm{~K}$ for building applications. Whereas the vapour pressure $p_{v}$ varies between zero at $0 \% \mathrm{RH}$ and $p_{\text {sat }}$ at $100 \% \mathrm{RH}$. Therefore the relative variation of $p_{v}$ can be considered much larger than the relative variation of $T$. That means, the pressure-driven diffusion exceeds the thermodiffusion by an order of magnitude. This had been expected from the thermodynamic approach.

\section{A.4 Micromechanical Approach for the Liquid Transport According to Whitaker}

Whitaker (1986a) gives a micromechanical approach for the liquid water transport. The Navier Stokes equation system for the advective flow in a Newton liquid is given by Stöcker (2004) p. 137:

$$
\begin{aligned}
\rho_{\ell} \cdot \frac{D v_{k}^{M_{\ell}}}{D t} & =-\frac{\partial}{\partial x_{k}} p_{\ell}+\rho_{\ell} \cdot g_{k}+\eta_{\ell} \cdot \Sigma_{j} \frac{\partial^{2}}{\partial x_{j}^{2}} v_{k}^{M_{\ell}} \\
\frac{D}{D t} & \equiv \frac{\partial}{\partial t}+\Sigma_{j}\left(v_{j}^{M_{\ell}} \frac{\partial}{\partial x_{j}}\right)
\end{aligned}
$$

In case of high friction the mass acceleration given on the left of (71) by the time derivative $D / D t\left(v_{k}^{M_{\ell}}\right)$ vanishes. This is the starting point of Whitaker (1986a), Eq. 2.2. He makes the 
following assumptions: Incompressibility of the liquid, Eq. 2.3:

$$
\vec{\nabla} \cdot \vec{v}^{M_{\ell}}=0
$$

Zero flow velocity at the boundary of the liquid phase, Eq. 2.4:

$$
\vec{v}^{M_{w}}\left(\vec{x} \in\left\{\partial V_{\ell}\right\}\right)=0
$$

The length scale of the pores is much smaller than the REV and this in turn is smaller than the macroscopic dimensions of the sample (Whitaker 1986a), Eq. 2.1:

$$
r_{\text {pore }}<<\ell_{\text {REV }}<<L_{\text {Sample }}
$$

Additionally, Whitaker assumes throughout his article, that important micromechanical properties like material porosity, liquid water density, viscosity and surface tension are not dependent on the system state, cf. (52).

From these assumptions, Darcy's law is derived (Whitaker 1986a), Eq. 3.38:

$$
\vec{v}^{M_{\ell}}=\underbrace{\frac{\theta_{\ell} \cdot \mathbf{C}^{-1}}{\eta_{\ell}}}_{K_{\ell}} \cdot\left(\vec{\nabla} p_{\ell}-\rho_{\ell} \cdot \vec{g}\right)
$$

The only driving force for the liquid water flow is the gradient of the liquid pressure $\vec{\nabla} p_{\ell}$ enhanced by the gravity force $\vec{g}$. Especially due to the assumptions (52) there is no thermodiffusion, i.e. no term containing $\vec{\nabla} T$. The conductivity $K_{\ell}$ is proportional to the liquid water content $\theta_{\ell}$ and inverse proportional to the viscosity $\eta_{\ell}$. The matrix $C$ describes the influence of the pore structure. It is given by (Whitaker 1986a), Eq. 3.30, 3.31, 3.35:

$$
\begin{aligned}
C & =-\frac{1}{V_{\ell}} \int_{\partial V_{\ell}} \vec{n}_{\partial V_{\ell}} \cdot(\vec{\nabla} \mathbf{B}-\mathbf{I} \vec{b}) \\
\vec{v}_{\text {micro }}^{M_{\ell}} & =\mathbf{B} \cdot \vec{v}^{M_{\ell}} \\
\vec{p}_{\text {micro }}^{M_{\ell}} & =\eta_{\ell} \cdot \vec{b} \cdot \vec{v}^{M_{\ell}}
\end{aligned}
$$

This is an integral over the deviations of the microscopic velocities/pressures from the velocities/pressures averaged over the REV, i.e. it depends on the shape of the velocity and pressure profiles due to the pore structure.

Whitaker extends his approach also to two-phase-flow (Whitaker 1986b) and to quadratic terms, the so-called "Forchheimer" terms (Whitaker 1996).

The explicit determination of $K$ according to (76) requires solving a differential equation system in the microscopic material. In fact, it could be easier to solve the equation system (71) directly. Quenard et al. (1998) and Bentz et al. (2000) tried to determine in this way the liquid moisture conductivity of a brick. The microstructure was determined by the evaluation of microscopic cross sections and by X-ray tomography. However, the procedure is quite difficult and reproduces the measured moisture conductivity only by order of magnitude.

\section{References}

Bednar, T.: Beurteilung des feuchte- und wärmetechnischen Verhaltens von Bauteilen und Gebäuden. Dissertation, Technical University Vienna (2000)

Bentz, D.P., Quenard, D.A., Kuenzel, H.M., Baruchel, J., Peyrin, F., Martys, N.S., Garboczi, E.J.: Microstructure and transport properties of porous building materials. II: three-dimensional X-ray tomographic studies.. Mater. Struct. 33(227), 147-153 (2000) 
Callen, H.B.: Thermodynamics and an Introduction to Thermostatistics. Wiley, New York (1985)

COMSOL: COMSOL Multiphysics. COMSOL, Stockholm (2005)

Darcy, H.: Les fontaines publiques de la ville de Dijon: exposition et application des principes a suivre et des formules a employer dans les questions de distribution d'eau. Dalmont, Paris (1856)

De Groot, S.R., Mazur, P.: Nonequilibrium Thermodynamics. Amsterdam (1962)

EN_ISO_13788: Hygrothermal performance of building components and building elements - Internal surface temperature to avoid critical surface humidity and interstitial condensation - Calculation methods (2001)

Funk, M., Grunewald, J., Nicolai, A.: Incorporation of salt related effects into a hygrothermal transport model. Paper accepted for the International Building Physics Conference, Concordia University Montreal, Canada, 27-31 August 2006

Gerthsen, C., Meschede, D., Vogel, H.: Gerthsen Physik. Springer, Berlin (2006)

Gray, W.G., Hassanizadeh, S.M.: Unsaturated flow including interface phenomena. Water Resour. Res. 27(8), 1855-1863 (1991)

Grunewald, J.: Diffusiver und konvektiver Stoff- und Energietransport in kapillarporösen Baustoffen. Dissertation, Technical University Dresden, Dresden (1997)

Grunewald, J.: Documentation of the Numerical Simulation program DIM3.1: Theoretical Fundamentals. Technical University Dresden (2000). Available via: http://bauklimatik-dresden.de/software.html

Grunewald, J., Nicolai, A.: CHAMPS-BES manual. Syracuse, NY (2006) Available at http:/beesl.syr.edu/champs.htm

Hagentoft, C.E. et al.: Assessment method of numerical prediction models for combined heat, air and moisture transfer in building components: benchmarks for one-dimensional cases. J. Therm. Envelope Build. Sci. 27(4), 327-352 (2004)

Hassanizadeh, S.M., Gray, W.G.: Mechanics and thermodynamics of multiphase flow in porous media including interface boundaries. Adv. Water Res. 13(4), 169-186 (1990)

Holm, A.: Ermittlung der Genauigkeit von instationären hygrothermischen Bauteilberechnungen mittels eines stochastischen Konzeptes. Dissertation, University Stuttgart (2001)

Ingham, D.B.: In: Popp, I. (ed.) Transport Phenomena in Porous Media. Pergamon, Oxford (1998)

Jarvis, NJ.: The MACRO model, Version 3.1. Technical descriptions and sample simulations. Uppsala (1994). Available via http://bgf.mv.slu.se/ShowPage.cfm?OrgenhetSida_ID=5658

Jaynes, E.T.: The minimum entropy production principle. Ann. Rev. Phys. Chem. 31, 579-601 (1980)

Künzel, H.M.: Verfahren zur ein- und zweidimensionalen Berechnung des gekoppelten Wärme- und Feuchtetransports in Bauteilen mit einfachen Kennwerten. Dissertation, Stuttgart University (1994)

Künzel, H.M.: Simultaneous heat and moisture transport in building components. Fraunhofer IRB Verlag, Stuttgart (1995)

Nicolai, A., Grunewald, J., Zhang, J.S.: Salztransport und Phasenumwandlung-Modellierung und numerische Lösung im Simulationsprogramm Delphin 5. Bauphysik 3, 231-239 (2007)

Pedersen, C.R.: Prediction of moisture transfer in building constructions. Build. Environ. 27(3), 387-397 (1992)

prEN_15026. Hygrothermal performance of building components and building elements - assessment of moisture transfer by numerical simulation (2006)

Quenard, D.A., Xu, K., Kunzel, H.M., Bentz, D.P., Martys, N.S.: Microstructure and transport properties of porous building materials. Mater. Struct. 31(209), 317-324 (1998)

Reif, F.: Fundamentals of Statistical and Thermal Physics. McGraw-Hill, Auckland (1985)

Rouquerol, F., Rouquerol, J., Sing, K.: Adsorption by Powders and Porous Solids. Academic Press, San Diego, CA (1999)

Sasic Kalagasidis, A.: HAM-tools. An integrated simulation tool for heat, air and moisture transfer analyses in building physics. Dissertation, Chalmers University of Technology, Göteborg (2004)

Scheffler, G., Grunewald, J., Haeupl, P.: Kalibrierung eines Ingenieurmodells zur hygrothermischen Materialcharakterisierung. Bauphysik 27, 191-195 (2005)

Simunek, J., Jarvis, N.J., van Genuchten, M.T., Gardenas, A.: Review and comparison of models for describing nonequilibrium and preferential flow and transport in the vadose zone. J. Hydrol. 272(1-4), 14-35 (2003)

Stöcker, H.: Taschenbuch der Physik: Formeln, Tabellen, Übersichten. Verlag Harri Deutsch, Frankfurt am Main (2004)

Whitaker, S.: Flow in porous media 1. A theoretical devolopment of Darcy's law. Transp. Porous Med. 1(1), 3-25 (1986a)

Whitaker, S.: Flow in porous media 2. The governing equations for immiscible flow. Transp. Porous Med. 1(2), 105-125 (1986b)

Whitaker, S.: The Forchheimer equation: a theoretical development. Transp. Porous Med. 25(1), 27-61 (1996) 